\title{
CD146 as an adverse prognostic factor in uterine sarcoma
}

Yun Zhou ${ }^{1,2,3 \dagger}$, He Huang ${ }^{1,2,3 \dagger}$, Lin-Jing Yuan ${ }^{1,2,3}$, Ying Xiong ${ }^{1,2,3}$, Xin Huang ${ }^{1,2,3}$, Jia-Xin Lin 1,2,3 and Min Zheng ${ }^{1,2,3^{*}}$

\begin{abstract}
Background: Uterine sarcoma is an aggressive malignancy with a poor prognosis. This study aimed to determine the expression of CD146, P53, and Ki-67 in uterine sarcoma and to evaluate their prognostic significance.

Methods: We retrospectively analyzed the prognosis and clinicopathologic features of 68 patients with uterine sarcoma. Immunohistochemical analyses of CD146, P53, and Ki-67 were performed in tissue samples collected from these patients and their relationship with prognosis was investigated.

Results: The 5-year overall survival (OS) rate was $46 \%$. Endometrial stromal sarcoma (ESS) patients had a better prognosis than leiomyosarcoma (LMS) patients, with a 2-year survival rate of $82 \%$. The membrane and cytoplasm of tumor cells exhibited CD146 overexpression in 8 (32\%) ESS cases, which was less than the 25 (69.4\%) cases observed in LMS and 2 (28.6\%) in MMMT. CD146 overexpression in the membrane and cytoplasm of tumor cells was closely related to lymph node metastasis $(P=0.021)$ and Ki-67 overexpression $(P=0.0053)$; there was no significant correlation with age, tumor size, International Federation of Obstetrics and Gynecology stage, or P53 overexpression in LMS.
\end{abstract}

Conclusions: CD146, P53, and Ki-67 are overexpressed in uterine sarcoma. CD146 expression correlates with lymph node metastasis and is associated with poor OS in LMS; it may be a potential prognostic marker for LMS.

Keywords: Uterine sarcoma, CD146 overexpression, Prognostic marker

\section{Background}

Uterine sarcoma, a rare and heterogeneous malignant tumor, accounts for only $3 \%$ of all uterine malignancies [1]; the annual incidence is $<2$ per 100,000 women [2]. According to the World Health Organization (WHO) 2003 classification system, there are three main uterine sarcoma subtypes: leiomyosarcoma (LMS), endometrial stromal sarcoma (ESS), and undifferentiated sarcoma (UD) [3]. Carcinosarcoma (malignant mixed Müllerian tumor, MMMT) is an undifferentiated sarcoma that was excluded as a uterine sarcoma in the 2009 National Comprehensive Cancer Network guidelines; however, most retrospective studies still include MMMT as a uterine

\footnotetext{
*Correspondence: zhengmin@sysucc.org.cn

${ }^{\dagger}$ Yun Zhou and He Huang are joint first authors and contributed equally to this work

${ }^{3}$ Collaborative Innovation Center for Cancer, 651 Dongfeng Road East, Guangzhou 510060, Guangdong, People's Republic of China

Full list of author information is available at the end of the article
}

sarcoma [4]. The prognostic factors of uterine sarcoma are not well established, with no consensus on their significance.

P53 gene mutations occur in approximately one-half of all tumors in a wide range of human malignancies and overexpression is associated with poor prognosis [5]. There are several reports of P53 overexpression in uterine LMS, MMMT, and ESS [6, 7]. Ki-67 expression is also associated with poor prognosis in uterine sarcoma [8]. During the last decade, several studies were performed to assess estrogen receptor, progesterone receptor, P53, and Ki-67 expression as prognostic factors in LMS [9].

CD146, an adhesion molecule belonging to the immunoglobulin superfamily, was first identified as a melanoma-specific cell adhesion molecule [10]. CD146 is overexpressed in cancers such as epithelial ovarian cancer and breast cancer, in addition to melanoma $[11,12]$. A recent study suggested that CD146 might be involved 
in tumor development and be associated with prognosis in several systemic tumors [13].

Previous studies have shown that CD146 is a tumor angiogenesis marker. Zhang et al. reported that CD146 is present in most blood vessels in cervical and endometrial cancer, suggesting that it may be actively involved in cervical and endometrial cancer metastasis via the vascular system [14]. The anti-CD146 monoclonal antibody AA98 inhibits angiogenesis and tumor growth. AA98 has antiangiogenic properties in vitro and in vivo. AA98 inhibits human umbilical vein endothelial cell proliferation and migration as well as angiogenesis, validating CD146 as a novel target for antiangiogenic agents [15]. Wen et al. found that CD146 expression promoted epithelial-mesenchymal transition, correlating with mesenchymal marker expression and indicating poor prognosis in gastric cancer. CD146 downregulation induced downregulation of the genes for vimentin, fibronectin, and thrombospondin, which are mesenchymal markers [16].

However, no study to date has reported on CD146 expression in uterine sarcoma. In the present study, we compiled data from patients diagnosed with uterine sarcoma from the Sun Yat-Sen University Cancer Center (SYSUCC) in Guangzhou, China to investigate associations between the expression of CD146, the cell proliferation molecule Ki-67, and P53 and the patients' clinicopathologic features and prognosis.

\section{Methods}

\section{Patient selection and treatment}

This retrospective study involved 68 patients diagnosed with primary uterine sarcoma who underwent surgical treatment from January 1996 to December 2011 at SYSUCC. Paraffin-embedded (PE) tissue samples were collected from the pathology department as stored specimens. Prior consent from patients was obtained for research use of their PE tissues. This study was approved by the SYSUCC Institutional Review Board. We agreed to provide copies of the appropriate documentation if requested.

Patients were examined at 2-month intervals for the first 2 years, at 6-month intervals for the next 3 years, and once per year thereafter. The median follow-up period was 63.3 months (range 1-272.1 months). The primary end point was any cancer-related death. To ensure diagnostic accuracy, experienced pathologists conducted the pathologic diagnoses. Clinical data were retrieved from medical records in the institutional database. The followup data were updated in August 2013.

The patients were selected using the following criteria: diagnosed with uterine sarcoma according to the 2003 WHO diagnostic criteria; no preoperative antitumor treatment; no previous malignant disease or second primary tumor; and complete clinicopathologic and follow-up data available. Patients who met these criteria were included.

The patients' clinicopathologic characteristics were: age at diagnosis; International Federation of Obstetrics and Gynecology (FIGO) stage; histopathologic subtype; surgical procedure; postoperative adjuvant therapy; pelvic lymphadenectomy; lymph node involvement; tumor size; distant metastasis and recurrence; and CD146, P53, and Ki-67 immunohistochemical staining patterns.

The standard surgical treatment for ESS and LMS was total abdominal hysterectomy (TAH) plus bilateral salpingo-oophorectomy (BSO); pelvic lymphadenectomy (PL) was performed for late-stage patients (stage III/ IV). Para-aortic lymph node dissection was performed for patients with suspected common iliac or para-aortic lymph node involvement. Omentectomy was performed for stage III/IV patients. Adjuvant radiotherapy and/ or adjuvant chemotherapy was administered to patients with the following pathologic risk factors: incomplete resection in late-stage cases; positive pelvic lymph nodes; vascular and lymphatic permeation; high-grade ESS; tumor size $>5 \mathrm{~cm}$; and mitotic index $(\mathrm{MI})>10 /$ highpower field (HPF). Radiotherapy comprised external pelvic irradiation (18 MV X-rays) using the multiport technique. Daily fractions of 1.8-2.0 Gy were administered over 5-6 weeks for a total dose of 50 Gy. Chemotherapy mainly comprised cisplatin $\left(60-75 \mathrm{mg} / \mathrm{m}^{2}, \mathrm{~d} 1\right)$ plus adriamycin $\left(40-50 \mathrm{mg} / \mathrm{m}^{2}, \mathrm{~d} 1\right)$ plus dacarbazine $\left(200 \mathrm{mg} / \mathrm{m}^{2}, \mathrm{~d} 1-5\right)$ as the primary drugs and was administered in three to five courses over a 3-week period.

The surgical treatment for MMMT usually comprised $\mathrm{TAH}$ plus BSO, PL, and omentectomy. We administered radiotherapy and chemotherapy for all MMMT cases.

\section{Immunohistochemistry}

Formalin-fixed and PE tumor tissue samples were used for immunohistochemical analysis. Sections ( $4 \mu \mathrm{m}$ thick) containing the area of the tumor with the highest grade of atypia and the least necrosis were obtained from the paraffin block, mounted on slides, and deparaffinized in xylene, ethanol, and distilled water. Following 10-min incubation with $3 \%$ hydrogen peroxide in methanol, the slides were boiled in citrate buffer solution (pH 6) for 10 min using a domestic pressure cooker. The sections were incubated overnight at $4{ }^{\circ} \mathrm{C}$ with P53, P27, Ki-67, and CD146 antibodies (1:100; Zhongshan Golden Bridge Biotechnology, Beijing, China). Then, the slides were washed with phosphate-buffered saline and incubated for $30 \mathrm{~min}$ at $37{ }^{\circ} \mathrm{C}$ with biotinylated goat antimouse secondary antibody (1:25; Lab Vision, Fremont, CA, USA). All slides were stained with 3,3'-diaminobenzidine and scored for immunoreactivity based on the percentage of positive tumor cells (percent positivity) and staining intensity (weak, 
moderate, strong). Slides with scores of $(-)$ or $(+)$ were deemed negative; slides with scores of $(++)$ or $(+++)$ were deemed positive. Two experienced pathologists confirmed the results in a double-blind analysis [12].

\section{Statistical analysis}

Statistical analyses were performed using SPSS v16.0 software (IBM, Armonk, NY, USA). Prognosis was determined based on overall survival (OS) and diseasefree survival (DFS). OS was calculated from the time of histopathological diagnosis to the time of death or last recorded event. DFS was calculated from the time of surgical resection to the first evidence of recurrence or death from any cause. Life tables were used to calculate the survival rates and median survival time. The relationship between CD146 expression and clinicopathologic characteristics was assessed using Pearson's Chi-square test. Survival curves were obtained using the Kaplan-Meier method. Log-rank tests were used for statistical comparison of curves. Univariate and multivariate Cox regression methods were adapted to evaluate potential prognostic factors, yielding hazard ratios and $95 \%$ confidence intervals. $P<0.05$ was considered statistically significant.

\section{Results}

\section{Demographic characteristics}

Table 1 summarizes the demographic characteristics of the patients. The mean age was 48.3 years; the

Table 1 Patients' clinical and histopathological characteristics $(n=68)$

\begin{tabular}{|c|c|c|c|c|}
\hline Characteristics & No. case & LMS $(n=36)$ & $\operatorname{ESS}(n=25)$ & MMMT $(n=7)$ \\
\hline \multicolumn{5}{|l|}{ Age (years) } \\
\hline$<50$ & $38(55.8 \%)$ & 20 & 17 & 1 \\
\hline$\geq 50$ & 30 (44.2\%) & 16 & 8 & 6 \\
\hline \multicolumn{5}{|l|}{ Stage } \\
\hline । & $36(53.9 \%)$ & 19 & 13 & 4 \\
\hline$\|$ & $3(4.4 \%)$ & 3 & 0 & 0 \\
\hline III & $22(32.4 \%)$ & 7 & 12 & 3 \\
\hline IV & $7(10.3 \%)$ & 7 & 0 & 0 \\
\hline \multicolumn{5}{|l|}{ Tumor size (cm) } \\
\hline$<5$ & 30 (44.1\%) & 16 & 11 & 3 \\
\hline$\geq 5$ & 38 (55.9\%) & 20 & 14 & 4 \\
\hline \multicolumn{5}{|l|}{ Pelvic lymphadenectomy } \\
\hline Yes & $34(50 \%)$ & 17 & 13 & 4 \\
\hline No & $34(50 \%)$ & 19 & 12 & 3 \\
\hline \multicolumn{5}{|l|}{ Lymph node metastasis } \\
\hline Positive & $24(35.3 \%)$ & 18 & 4 & 2 \\
\hline Negative & $44(64.7 \%)$ & 33 & 9 & 2 \\
\hline \multicolumn{5}{|l|}{ Surgical procedure } \\
\hline $\mathrm{TAH} \pm \mathrm{BSO} / \mathrm{OT} / \mathrm{PL} / \mathrm{PLND}$ & $8(11.8 \%)$ & 4 & 4 & 0 \\
\hline $\mathrm{TAH}+\mathrm{BSO} \pm / \mathrm{PL} / \mathrm{OT}$ & $29(42.6 \%)$ & 17 & 12 & 0 \\
\hline $\mathrm{TAH}+\mathrm{BSO}+\mathrm{PL} \pm \mathrm{OT} \pm \mathrm{PLND}$ & $31(45.6 \%)$ & 15 & 9 & 7 \\
\hline Chemotherapy & $22(32.4 \%)$ & 13 & 9 & 0 \\
\hline Radiotherapy & $4(5.9 \%)$ & 1 & 3 & 0 \\
\hline Hormone therapy & $1(1.5 \%)$ & 0 & 1 & 0 \\
\hline Chemotherapy and radiotherapy & $20(29.4 \%)$ & 10 & 3 & 7 \\
\hline Chemotherapy and hormone therapy & $3(4.4 \%)$ & 1 & 2 & 0 \\
\hline Radiotherapy and hormone therapy & $1(1.5 \%)$ & 0 & 1 & 0 \\
\hline No adjuvant therapy & $17(25 \%)$ & 11 & 6 & 0 \\
\hline \multicolumn{5}{|l|}{ Distant metastasis and recurrence } \\
\hline Pelvic & $10(14.7 \%)$ & 6 & 3 & 1 \\
\hline Pelvic and liver/lung & $6(8.8 \%)$ & 4 & 2 & 0 \\
\hline Lung & $4(5.9 \%)$ & 3 & 1 & 0 \\
\hline Other parts & $6(8.8 \%)$ & 5 & 1 & 0 \\
\hline None & 42 (61.5\%) & 18 & 18 & 6 \\
\hline
\end{tabular}

TAH total abdominal hysterectomy, BSO bilateral salpingo-oophorectomy, OT omentectomy, PL pelvic lymphadenectomy, PLND para-aortic lymph node dissection 
median age was 47.5 years (range $20-81$ years). Primary symptoms included irregular menstrual bleeding (19.2\%), postmenopausal or abnormal vaginal bleeding (30.8\%), pelvic mass (11.5\%), and abdominal pain $(15.4 \%)$. Twelve cases $(17.6 \%)$ were diagnosed incidentally. Of the 68 patients, 36 (52.9\%) had LMS, 25 (36.8 \%) had malignant ESS, and 7 (10.3 \%) had MMMT. Thirty-six cases (52.9\%) were stage I, 3 (4.4\%) were stage II, 22 (32.4\%) were stage III, and 7 (10.3\%) were stage IV.

All patients underwent surgery as initial treatment: 8 (11.8 \%) underwent TAH, 29 (42.6\%) TAH + BSO, and $31(45.6 \%)$ TAH + BSO + PL. Fifty-one patients (75\%) received postoperative adjuvant therapy. Lymphovascular space invasion (LVSI) and lymph node metastasis were observed in 24 patients (35.3\%). Twenty-six patients (38.2 \%) developed recurrence and 25 patients (36.8\%) died during follow-up.

\section{CD146, P53, and Ki-67 expression and their relationship with clinicopathologic characteristics of uterine sarcoma} On immunohistochemical analysis of the uterine sarcoma tissue specimens, CD146-positive staining was localized not only in the epithelial compartment (the membrane and cytoplasm of tumor cells) but also in the vascular compartment. CD146 expression occurred more often in the vascular compartment. Forty-nine cases (72\%) exhibited vascular compartment CD146 expression, 35 of which were also positive for CD146 staining in the epithelial compartment (Fig. 1).

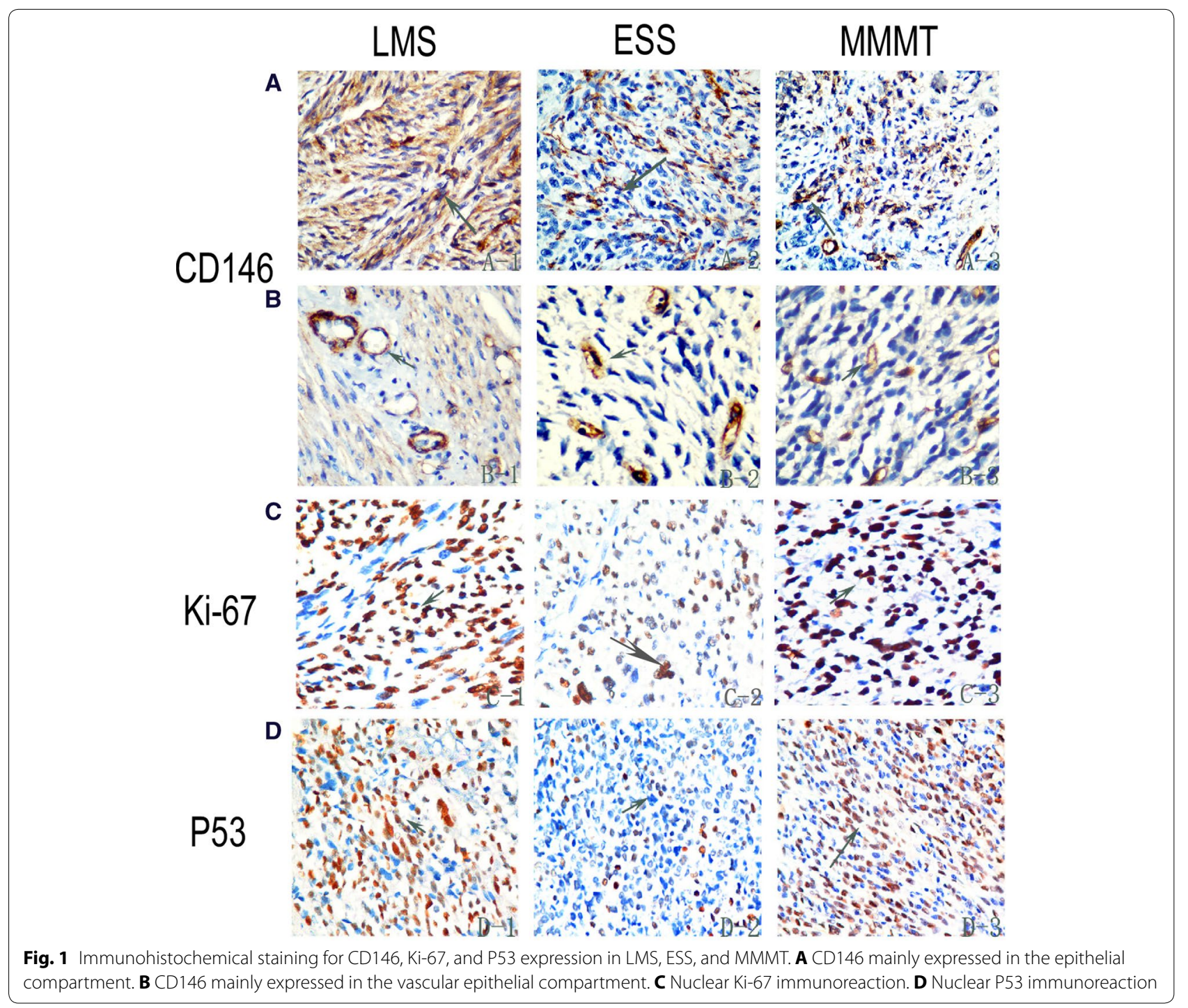


Overexpression of CD146 in the membrane and cytoplasm of tumor cells was observed in 8 (32\%) ESS cases, which was less than the 25 (69.4\%) cases observed in LMS and 2 (28.6\%) in MMMT. Positive nuclear P53 and Ki-67 immunoreactions were observed in 30 (44.1\%) and 48 (70.6 \%) cases, respectively. ESS patients had the lowest positivity rates for both P53 and Ki-67 immunoreaction, with P53 and Ki-67 expressed in seven (28\%) and six $(24 \%)$ cases, respectively (Table 2 ).

We analyzed the correlation between CD146 expression status and the clinicopathological characteristics of LMS; the results are summarized in Table 3. CD146 overexpression on membrane and cytoplasm staining was closely related to lymph node metastasis $(P=0.021)$ and Ki-67 overexpression $(P=0.0053)$, but there was no

Table 2 Immunohistochemical analysis of uterine sarcoma specimens

\begin{tabular}{lllll}
\hline & CD146 $^{\mathbf{a}}$ & CD146 $^{\mathbf{b}}$ & P53 & Ki67 \\
\hline LMS & $25 / 36$ & $32 / 36$ & $22 / 36$ & $24 / 36$ \\
ESS & $8 / 25$ & $12 / 25$ & $7 / 25$ & $6 / 25$ \\
MMMT & $2 / 7$ & $5 / 7$ & $1 / 7$ & $4 / 7$ \\
\hline
\end{tabular}

ESS endometrial stromal sarcoma, LMS leiomyosarcoma, MMMT malignant mixed Müllerian tumor

a Cytoplasm and membrane staining

b Vascular endothelial cell staining

Table 3 Correlation between CD146 expression and clinicopathologic features in LMS

\begin{tabular}{|c|c|c|c|}
\hline & CD146 (-) & CD146 (+) & $P$ value \\
\hline \multicolumn{4}{|l|}{ Age } \\
\hline$<50$ & 6 & 12 & \multirow[t]{2}{*}{0.6} \\
\hline$\geq 50$ & 5 & 13 & \\
\hline \multicolumn{4}{|l|}{ Stage } \\
\hline$|-| \mid$ & 7 & 11 & \multirow[t]{2}{*}{0.4} \\
\hline III-IV & 4 & 14 & \\
\hline \multicolumn{4}{|c|}{ Tumor size (cm) } \\
\hline$<5$ & 14 & 8 & \multirow[t]{2}{*}{0.16} \\
\hline$\geq 5$ & 12 & 17 & \\
\hline \multicolumn{4}{|l|}{ LN statue } \\
\hline Positive & 2 & 15 & \multirow[t]{2}{*}{0.021} \\
\hline Negative & 9 & 10 & \\
\hline \multicolumn{4}{|c|}{ P53 expression } \\
\hline Positive & 7 & 15 & \multirow[t]{2}{*}{0.68} \\
\hline Negative & 4 & 10 & \\
\hline \multicolumn{4}{|c|}{ Ki67 expression } \\
\hline Positive & 4 & 20 & \multirow[t]{2}{*}{0.0053} \\
\hline Negative & 8 & 5 & \\
\hline
\end{tabular}

significant correlation with age, tumor size, FIGO stage, or P53 overexpression.

\section{Relationship between clinicopathologic parameters and survival}

The median survival time was 30.7 months (range 1-271 months). The 2- and 5-year OS rates were 57 and $46 \%$, respectively; the 2- and 5-year DFS rates were 51 and $44 \%$, respectively.

Even in more advanced-stage patients, the ESS group had better OS and DFS than the LMS group (Fig. 2A). The 2-year OS rate for ESS and LMS patients was 82 and $66 \%$, respectively. The median OS for ESS and LMS patients was 136.5 and 61.02 months, respectively $(P=0.024)$. The median DFS for ESS and LMS patients was 135.6 and 24.6 months, respectively $(P=0.037)$.

The median survival of patients with lymph node metastasis was 57 months compared with 136 months in lymph node-negative patients $(P<0.05)$. Tumor size $\geq 5 \mathrm{~cm}$ was a significant adverse prognostic factor for LMS $(P=0.02)$. The median survival of patients with tumor size $<5$ or $\geq 5 \mathrm{~cm}$ was 100 and 40 months, respectively. MI $>10 / \mathrm{HPF}$ tended to be associated with an unfavorable outcome in LMS $(P=0.1)$. Multivariate analyses showed no statistical differences among these factors.

Adjuvant therapy had little impact on the management of uterine sarcoma, especially in early-stage disease. Postoperative chemotherapy did not improve the survival time of stage I ESS patients $(P=0.42)$.

\section{Prognostic significance of CD146, P53, and Ki-67 expression}

CD146-positive LMS patients had a poorer prognosis than those who were CD146 negative. The 2-year OS rate was $53 \%$ for CD146-positive patients and $84 \%$ for CD146-negative patients $(P=0.0039$, Fig. 2B). On multivariate analyses, CD146 positivity varied statistically significantly with clinicopathological parameters, P53, and Ki-67 expression $(P=0.01)$.

Ki-67 overexpression was also related to an unfavorable outcome in LMS, with a significant difference observed $(P=0.0063$, Fig. 2 C). P53 overexpression in LMS showed no significant difference $(P=0.1)$.

In LMS patients with tumor size $\geq 5 \mathrm{~cm}$, Kaplan-Meier estimates yielded a significant survival difference favoring CD146-positive patients ( $P=0.0086$, Fig. 2D).

CD146 positivity also tended to be associated with poor prognosis in ESS, though the difference was not significant $(P=0.1)$. Similarly, P53 and Ki-67 overexpression in ESS were related to poor prognosis but the differences were not significant $(P=0.1)$. 

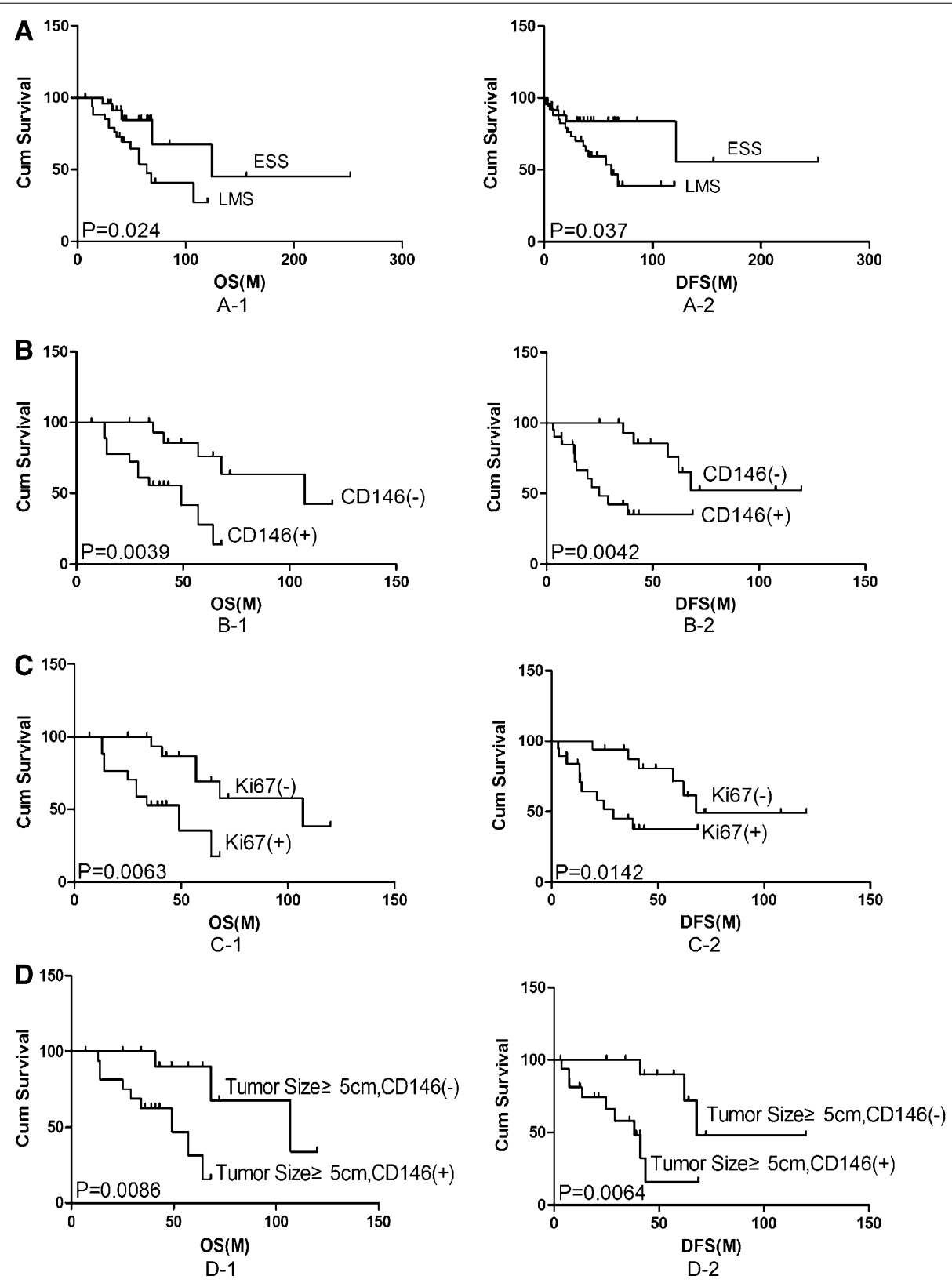

Fig. 2 Kaplan-Meier curves obtained from survival analyses (log-rank) of patients with uterine sarcoma. A Histologic type was a significant prognostic factor for overall survival in LMS and ESS $(P=0.024)$. B CD146 showing significant correlation with overall survival in LMS. CD146-positive patients had a poor prognosis compared with CD146-negative patients $(P=0.0039)$. C Ki67 showing significant correlation with overall survival in LMS. Ki67-positive patients had a poor prognosis compared with Ki67-negative patients $(P=0.0063)$. D CD146 expression may be correlated with tumor size and poor prognosis in LMS $(P=0.026)$

\section{Discussion}

Uterine sarcomas display aggressive clinical behavior and have a poor prognosis [3]. Our findings are in line with another study [17] in that the most common histologic type of uterine sarcoma detected was LMS (52.9\%) whereas MMMT accounted for only $10.3 \%$ of cases. The median survival time of the 68 patients was 30.7 months; the 5-year OS and DFS rates were 46 and $44 \%$, respectively, which is consistent with a previous report in which OS and DFS were $45-47$ and 34-36\%, respectively [17]. In a previous study, the outcome of ESS was significantly better than that of LMS and MMMT [18]. However, other studies have found, via multivariate analyses, that the histologic subtype does not affect survival. In our 
study, ESS patients had a better prognosis than those with other histologic subtypes. Even in more advancedstage patients, the ESS group had better OS and DFS than the LMS group, with a 2-year survival rate of $82 \%$. A possible reason is that ESS tends to present as a less aggressive, early-stage disease with low levels of metastasis [18].

Previous studies have found that stage, surgical treatment, tumor grade, tumor size, and MI are prognostic factors for uterine sarcoma $[19,20]$. Our results suggest that tumor size $\geq 5 \mathrm{~cm}$, lymph node metastasis, and MI are significant prognostic factors in LMS. In our study, 38 of 68 cases (55.9\%) of primary uterine sarcoma expressed CD146 in the epithelial compartment. In LMS, CD146 expression in the epithelial compartment was associated with lymph node metastasis and was related to poor OS. Conversely, CD146 positivity in ESS patients did not correlate with OS. CD146-positive LMS had a poorer prognosis, the 2-year OS rate being $53 \%$ compared with the 2 -year OS of $84 \%$ in CD146-negative LMS $(P=0.0039)$. In LMS patients with tumor size $\geq 5 \mathrm{~cm}$, the outcome was worse for those who were CD146 positive $(P=0.0086)$. Similar to other carcinomas, CD146 was overexpressed and associated with tumor metastasis and poor prognosis $[11,21]$. Aldovini et al. demonstrated that CD146 is involved in ovarian cancer and is a marker for poor prognosis in this disease [21].

CD146 is a $113 \mathrm{kDa}$ integral membrane glycoprotein, the amino acid sequence of which comprises a signal peptide, an extracellular fragment structure of five immunoglobulin-like domains ( $\mathrm{V}-\mathrm{V}-\mathrm{C} 2-\mathrm{C} 2-\mathrm{C} 2)$, a transmembrane region, and a short cytoplasmic tail [22]. It has been suggested that CD146 promotes tumor growth, angiogenesis, and metastasis [10]. CD146 plays a critical pro-migratory role in the vascular system, normal development, and tumor progression patterning [23].

Interestingly, in the present study, CD146 was expressed in the vascular compartment in 49 cases, but also in the epithelial compartment in 38 cases. It has been established that LVSI is a significant prognostic factor in uterine sarcoma; LVSI and lymph node metastasis were shown to be relevant prognostic factors with an impact on OS and distant metastasis-free survival [24]. Moreover, on multivariate analysis, we found a significant relationship between CD146 overexpression and prognosis $(P=0.01)$. This implies that $C D 146$ overexpression in the epithelial compartment may be related to angiogenesis and metastasis with a worse prognosis in LMS, and may serve as a potential LMS prognostic marker.

The prognosis of MMMT and UD may be related to CD146 expression in the vascular compartment. Due to the limited number of cases, we were unable to obtain significant statistical differences. The correlation between
CD146 expression and clinicopathologic characteristics was not statistically significant.

CD146 positivity in the epithelial compartment showed a significant association with poor prognosis in ESS $(P=0.1)$. Likewise, P53 and Ki-67 overexpression in LMS were related to an unfavorable outcome, though no significant difference was observed $(P=0.1)$. One possible reason may be a lack of test power, as the sample size was small, or it could have been due to the different mechanism of progression and metastasis in ESS.

\section{Conclusions}

CD146, P53, and Ki-67 were overexpressed in uterine sarcoma. CD146 expression in the epithelial compartment was correlated with lymph node metastasis and was associated with poor OS in LMS, but had no impact on survival in ESS. CD146 may be a potential prognostic marker for LMS.

\section{Abbreviations}

WHO: World Health Organization; LMS: leiomyosarcoma; ESS: endometrial stromal sarcoma; UD: undifferentiated sarcoma; MMMT: malignant mixed Müllerian tumor; SYSUCC: Sun Yat-Sen University Cancer Center; FIGO: International Federation of Obstetrics and Gynecology; PE: paraffin-embedded; $\mathrm{TAH}$ : total abdominal hysterectomy; BSO: bilateral salpingo-oophorectomy; PL: pelvic lymphadenectomy; MI: mitotic index; HPF: high-power field; OS: overall survival; DFS: disease-free survival; LVSI: Iymphovascular space invasion.

\section{Authors' contributions}

$M Z, H H$, and $Y Z$ conceived of and designed the study; L-JY and $Y Z$ carried out immunohistochemistry experiments; $Y X$ and $X H$ participated in the design of the study; $\mathrm{HH}$ and $Y Z$ drafted the manuscript; J-XL and $Y Z$ performed the statistical analysis. All authors conceived of the study, and participated in its design and coordination and helped to draft the manuscript. All authors read and approved the final manuscript.

\section{Author details}

${ }^{1}$ Department of Gynecology Oncology, Sun Yat-sen University Cancer Center, Guangzhou, People's Republic of China. ${ }^{2}$ State Key Laboratory of Oncology in South China, Guangzhou, People's Republic of China. ${ }^{3}$ Collaborative Innovation Center for Cancer, 651 Dongfeng Road East, Guangzhou 510060, Guangdong, People's Republic of China.

\section{Acknowledgements}

This study was supported by the National Natural Science Foundation of China (Grant No. 81171948 and No. 81372275), the Key Program of Natural Science Foundation of Guangdong Province, China (Grant No. S2012020011060), and the Project of State Key Laboratory of Oncology in South China (Grant No. 030041060004).

Compliance with ethical guidelines

\section{Competing interests}

The authors declare that they have no competing interests.

Received: 14 December 2014 Accepted: 7 August 2015 Published online: 21 August 2015

\section{References}

1. Mundhenke C, Bauerschlag D, Fischer D, Friedrich M, Maass N (2007) Malignant tumors of the uterus. Ther Umsch 64:381-388 
2. Amant F, Coosemans A, Debiec-Rychter M, Timmerman D, Vergote I (2009) Clinical management of uterine sarcomas. Lancet Oncol 10:1188-1198

3. Park JY, Kim DY, Suh DS, Kim JH, Kim YM, Kim YT et al (2008) Prognostic factors and treatment outcomes of patients with uterine sarcoma analysis of 127 patients at a single institution. J Cancer Res Clin Oncol 134:1277-1287

4. Trope CG, Abeler VM, Kristensen GB (2012) Diagnosis and treatment of sarcoma of the uterus. a review. Acta Oncol 51:694-705

5. Tsao MS, Aviel-Ronen S, Ding K, Lau D, Liu N, Sakurada A et al (2007) Prognostic and predictive importance of p53 and RAS for adjuvant chemotherapy in non small-cell lung cancer. J Clin Oncol 25:5240-5247

6. Bur ME et al (1992) P53 expression in neoplasms of the uterine corpus. Am J Clin Pathol 98(1):81-87

7. Niemann TH et al (1995) P53 protein overexpression in smooth muscle tumors of the uterus. Hum Pathol 26(4):375-379

8. Zhai YL et al (1999) Expression of steroid receptors, Ki-67, and p53 in uterine leiomyosarcomas. Int J Gynecol Pathol 18(1):20-28

9. Mayerhofer K et al (2004) Ki-67 and vascular endothelial growth factor expression in uterine leiomyosarcoma. Gynecol Oncol 92(1):175-179

10. Lehmann JM, Riethmuller G, Johnson JP (1989) MUC18, a marker of tumor progression in human melanoma, shows sequence similarity to the neural cell adhesion molecules of the immunoglobulin superfamily. Proc Natl Acad Sci USA 86(24):9891-9895

11. Aldovini D et al (2006) M-CAM expression as marker of poor prognosis in epithelial ovarian cancer. Int J Cancer 119(8):1920-1926

12. Zabouo G et al (2009) CD146 expression is associated with a poor prognosis in human breast tumors and with enhanced motility in breast cancer cell lines. Breast Cancer Res 11(1):R1

13. Liu WF et al (2012) CD146 expression correlates with epithelial-mesenchymal transition markers and a poor prognosis in gastric cancer. Int J Mol Sci 13(5):6399-6406

14. Zhang $\mathrm{H}$ et al (2013) CD146 is a potential marker for the diagnosis of malignancy in cervical and endometrial cancer. Oncol Lett 5(4):1189-1194
15. Ouhtit A et al (2009) Towards understanding the mode of action of the multifaceted cell adhesion receptor CD146. Biochim Biophys Acta 1795(2):130-136

16. Yan X et al (2003) A novel anti-CD146 monoclonal antibody, AA98, inhibits angiogenesis and tumor growth. Blood 102(1):184-191

17. Zheng $\mathrm{M}$ et al (2010) Overexpression of karyopherin-2 in epithelial ovarian cancer and correlation with poor prognosis. Obstet Gyneco 116(4):884-891

18. Durnali A et al (2012) Prognostic factors and treatment outcomes in 93 patients with uterine sarcoma from 4 centers in Turkey. Asian Pac J Cancer Prev 13(5):1935-1941

19. Denschlag D et al (2007) Prognostic factors and outcome in women with uterine sarcoma. Eur J Surg Oncol 33(1):91-95

20. Kim SH et al (2006) Prognostic factors and expression of p53 and mdm-2 in uterine sarcomas. Int J Gynaecol Obstet 95(3):272-277

21. Major FJ et al (1993) Prognostic factors in early-stage uterine sarcoma. A Gynecologic Oncology Group study. Cancer 71(4 Suppl):1702-1709

22. Zeng Q et al (2012) CD146, an epithelial-mesenchymal transition inducer, is associated with triple-negative breast cancer. Proc Natl Acad Sci USA 109(4):1127-1132

23. Johnson JP, Rothbacher U, Sers C (1993) The progression associated antigen MUC18: a unique member of the immunoglobulin supergene family. Melanoma Res 3(5):337-340

24. Rovirosa A et al (2002) Is vascular and lymphatic space invasion a main prognostic factor in uterine neoplasms with a sarcomatous component? A retrospective study of prognostic factors of 60 patients stratified by stages. Int J Radiat Oncol Biol Phys 52(5):1320-1329

\section{Submit your next manuscript to BioMed Central and take full advantage of:}

- Convenient online submission

- Thorough peer review

- No space constraints or color figure charges

- Immediate publication on acceptance

- Inclusion in PubMed, CAS, Scopus and Google Scholar

- Research which is freely available for redistribution

Submit your manuscript at

www.biomedcentral.com/submit

C BioMed Central 